\title{
The Burden of Acute Febrile Illness Attributable to Dengue Virus Infection in Sri Lanka: A Single-Center 2-Year Prospective Cohort Study (2016-2019)
}

\author{
Hasitha Tissera, ${ }^{1}$ Preshila Samaraweera, ${ }^{2}$ Melanie de Boer, ${ }^{3 *}$ Sanjay Gandhi, ${ }^{4}$ Ludovic Malvaux,${ }^{5}$ Shailesh Mehta, ${ }^{6}$ \\ Paba Palihawadana, ${ }^{1}$ Valerie Vantomme,${ }^{5}$ Robert Paris, ${ }^{3}$ and Alexander Schmidt ${ }^{3}$ \\ ${ }^{1}$ Epidemiology Unit, Ministry of Health, Colombo, Sri Lanka; ${ }^{2}$ National Dengue Control Unit, Ministry of Health, Colombo, Sri Lanka; \\ ${ }^{3}$ GSK, Rockville, Maryland; ${ }^{4}$ GSK, Mumbai, India; ${ }^{5}$ GSK, Rixensart, Belgium; ${ }^{6}$ GSK, Wavre, Belgium
}

\begin{abstract}
We performed a 2-year prospective cohort study to determine the incidence of dengue in Angoda, Colombo district, Sri Lanka (NCT02570152). The primary objective was to determine the incidence of acute febrile illness (AFI) because of laboratory confirmed dengue (LCD). Secondary objectives were to determine AFI incidence because of non-LCD, describe AFI symptoms, and estimate AFI incidence because of LCD by dengue virus (DENV)-type and age group. Participants from households with at least one minor and one adult ( $\leq 50$ years) were enrolled and followed with scheduled weekly visits and, in case of AFI, unscheduled visits. Blood was collected for DENV detection at AFI visits, and symptoms recorded during the 7-day period following AFI onset. A total of 2,004 participants were enrolled (971 children, and 1,033 adults). A total of 55 LCD episodes were detected (overall incidence of 14.2 per 1,000 person-years). Incidence was the highest among children $<5$ years (21.3 per 1,000 person-years) and 5-11 years (22.7 per 1,000 person-years), compared with adults $\geq 18$ years (9.2 per 1,000 person-years). LCD was mostly (83.6\%) caused by DENV-2 $(n=46)$, followed by DENV-1 $(n=6)$ and DENV-3 $(n=3)$. Common symptoms of LCD were headache, fatigue, myalgia, loss of appetite, and arthralgia. Incidence of AFI because of non-LCD was 47.3 per 1,000 person-years. In conclusion, this study reports the LCD incidence for a DENV-2 dominated epidemic that is comparable to the incidence of suspected dengue reported passively for 2017 , one of the worst outbreaks in recent history.
\end{abstract}

\section{INTRODUCTION}

Dengue is an arboviral illness caused by one of four different serotypes of infecting virus (dengue virus [DENV]-1, DENV-2, DENV-3, and DENV-4). The main vector is Aedes aegypti, a mosquito that is highly adapted to urban environments. Symptoms of DENV infection include fever, headache, skin erythema, arthralgia, and myalgia, which are also common symptoms of other febrile illnesses; thus, misdiagnosis and underreporting are common. Dengue has three phases: a febrile phase, a critical phase, and a recovery phase, of which the febrile phase has a sudden onset and lasts about 2-7 days. The clinical manifestations have a wide range, from mild fever to more severe dengue hemorrhagic fever and potentially fatal dengue shock syndrome. ${ }^{1}$

Dengue is the most important mosquito-borne viral disease globally, of which the incidence has increased 30-fold during the past five decades. ${ }^{1}$ Geographically, half the world's population is at risk of DENV infection, and it was estimated that in 2013, globally, 58.4 (uncertainty interval 23.6-121.9) million people were infected. ${ }^{2}$ Although the disease's exact distribution is uncertain, dengue is found in tropical and subtropical climates, mostly in urban and semiurban areas. ${ }^{3}$ According to the World Health Organization (WHO), an estimated 100 countries are affected, ${ }^{4}$ although there is strong evidence for dengue occurrence in 128 countries. ${ }^{5}$ About $70.0 \%$ of the disease burden is in Asia. ${ }^{6}$

According to the WHO Global Strategy for Dengue Prevention and Control, improved surveillance is needed to estimate dengue disease burden better. ${ }^{4}$ Improved surveillance would benefit reporting, prevention, and control of dengue, whereas incidence rates and clinical data would also support

*Address correspondence to Melanie de Boer, GSK, 14200 Shady Grove Rd., Rockville, MD 20850. E-mail: melanie.ann.de.boer@ gmail.com the definition of clinical endpoints in future vaccine efficacy trials.

This prospective cohort study was designed to support the selection and definition of clinical and laboratory endpoints for future dengue vaccine efficacy trials and provide incidence data for sample size calculation in Sri Lanka. The study's primary objective was to determine the incidence of acute febrile illness (AFI) because of laboratory confirmed dengue (LCD) in the study population. Secondary objectives were to determine the incidence of AFI because of non-LCD, describe the signs and symptoms of $\mathrm{AFI}$, and estimate the incidence of AFI because of LCD by DENV-type and age group.

\section{METHODS}

Study design. This study was a prospective, communitybased, cohort study performed in an urban Kolonnawa Medical Officer of Health $(\mathrm{MOH})$ division in the Colombo district, Sri Lanka, by household sampling. The study period was from June 1, 2016 to June 28, 2019. Recruitment of the participants took place from June 1, 2016 to June 28, 2017 after which each participant was followed for 2 years. Follow-up of the last patient was completed on June 28, 2019. The study consisted of scheduled clinic visits, including the enrollment visit (Visit 1) and the close-out visit (Visit 2), and weekly contacts. The weekly contacts consisted of home visits at least every other week and telephone contacts. In addition, in the case of AFI, an unscheduled Suspected Dengue Visit, optional Return Visit, and Follow-up Visit were also performed. AFI was defined as body temperature $\geq 38^{\circ} \mathrm{C}$ on $\geq 2$ consecutive days, measured at least twice, and at least 8 hours apart. Blood samples were only collected during the Suspected Dengue Visit. The study design is illustrated in Figure 1.

In the event of AFI, blood samples were collected for participants $\geq 5$ years of age. For participants $<5$ years of age, 


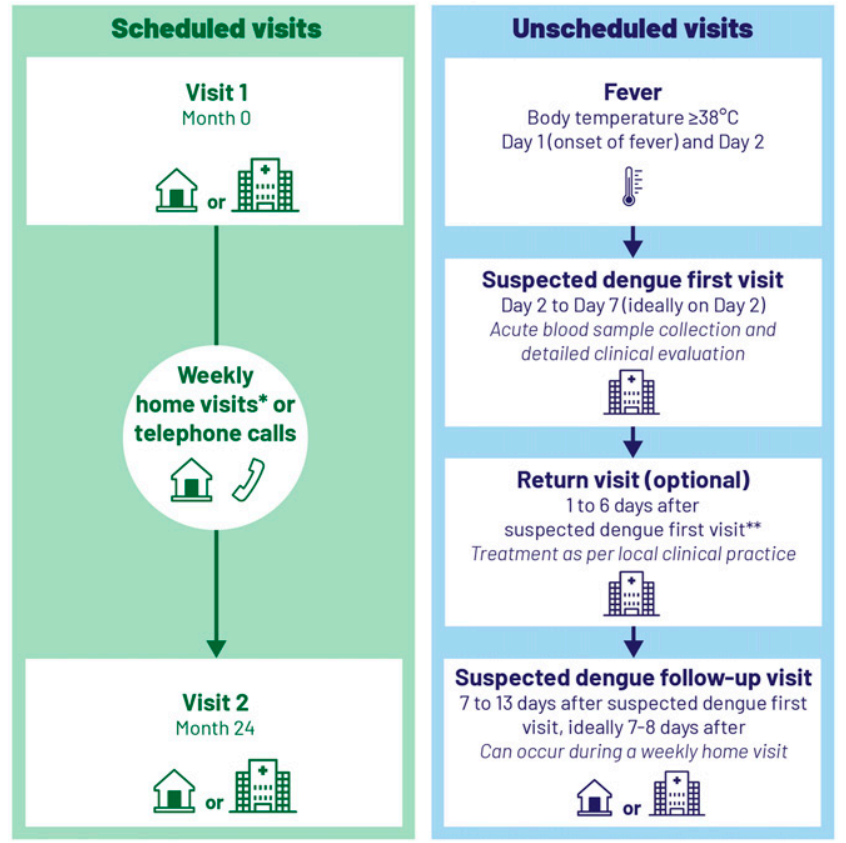

Figure 1. Study design. * At least every other week. ${ }^{*}$ As per investigator judgment. This figure appears in color at www.ajtmh.org.

samples were collected only if clinically indicated, as per investigator judgment. Depending on the patient's age, pediatric or adult sample collection kits were provided that included vials for dengue nonstructural protein 1 (NS1)-rapid antigen test, confirmatory reverse transcriptase-polymerase chain reaction (RT-PCR), and complete blood count. A total of $5 \mathrm{~mL}$ blood was collected into individual vials using the sampling kit identified with a unique number for each case and episode. A designated laboratory technician under the supervision of a specialist microbiologist/hematologist performed both NS1-rapid antigen test and complete blood count at the infectious disease hospital laboratory on the same day, while the serum samples for RT-PCR were stored at $-80^{\circ} \mathrm{C}$ at the hospital laboratory. Batches of samples were shipped monthly to a designated laboratory in the United Kingdom for RT-PCR testing and dengue virus serotyping. LCD was defined as AFI with laboratory confirmation of dengue by RT-PCR using the Simplexa Dengue kit (Focus Diagnostics, San Juan Capistrano, CA; Simplexa is a trademark owned by or licensed to Focus Diagnostics) on the acute serum sample taken during the 7-day period (Days 2-7) from the onset of fever.

The study was conducted following the International Council for Harmonization of Good Clinical Practice guidelines ${ }^{7}$ and the Declaration of Helsinki. The study protocol, amendments, and other study-related documents were reviewed and approved by the Ethics Review Committee of the Faculty of Medicine at the University of Colombo, Sri Lanka. The study was registered at clinicaltrials.gov (NCT02570152). ${ }^{8}$

Participants. The study population included participants living in randomly selected households in geographically defined communities at Kolonnawa, Colombo, Sri Lanka. In the Kolonnawa $\mathrm{MOH}$ division, six areas were selected randomly from a total of 46 smallest administrative areas. A list of randomly selected households was prepared according to probability-proportional-to-size sampling of the population using the census register in each of the six areas. Trained research assistants approached the households. Selected households were considered eligible for inclusion if at least one household member $\geq 6$ months to $<18$ years of age assented and at least one adult ( $\leq 50$ years) who consented for himself/herself and the minor in the household to participate in the study. The protocol targeted 2,000 participants to be enrolled.

The inclusion criteria involved 1) in the opinion of the investigator, the participant (or participant's parent[s]/legally acceptable representative [LAR]) and the minor were able to participate and would comply with the requirements of the protocol; 2) signed/thumb-printed informed consent (and assent if applicable) was obtained from the participant/participant's parent(s)/LAR(s); 3) the participant was part of a household with at least one child $(\geq 6$ months and $<18$ years of age) and at least one adult ( $\leq 50$ years of age) of which informed consent (and assent if applicable) was obtained; and 4) participants planned-at the time of enrollment-to remain in the same household during the study period.

The exclusion criteria involved: 1) child in care; 2) participation in another epidemiologic study or clinical trial that would conflict with the current study, based on the investigator's judgment; and 3) terminal illness or mental incapacity based on the investigator's judgment.

Statistical analysis. Descriptive statistics (mean, standard deviation [SD], and frequency) were calculated for sociodemographic characteristics. There was a single analysis set (total cohort) used in this study that included all participants enrolled. The incidence rate of first AFI because of LCD was calculated along with the $95 \%$ confidence interval $(\mathrm{Cl})$, based on the total cohort. The incidence rate of first AFI because of LCD by serotype (DENV-1 to -4 ) and incidence rate of all AFI because of non-LCD with $95 \%$ exact $\mathrm{Cl}$ were calculated for overall and age group, based on the total cohort.

A description of signs and symptoms of AFI because of LCD and because of non-LCD included the percentage of AFI presenting each sign or symptom (any intensity and grade 3) during the 7-day period from the onset of AFI. Duration of signs and symptoms were also described for LCD and non-LCD episodes. Analyses were performed, overall and separately for each age group. Univariate and multivariate stepwise logistic regressions were performed to investigate the association of signs and symptoms with AFI. Grading of symptoms is specified in the Supplemental Material (Supplemental Table 1).

We assumed a drop-out rate of $10.0 \%$ in the first year and a drop-out rate of $20.0 \%$ in the second year for the sample size calculation. If a maximum of 2,000 participants were enrolled for a 2-year follow-up period, the final analysis would be performed on approximately 3,300 person-years overall. Participants withdrawn or lost to follow-up were not replaced, and missing data were not imputed. Age was determined at the time of enrollment. The statistical analyses were performed using the Statistical Analysis System Version 9.4 (SAS Institute Inc., Cary, NC). 


\section{RESULTS}

Baseline characteristics. A total of 2,004 participants (total cohort) were enrolled. About half of the participants were adults $18-50$ years of age $(n=1,033 ; 51.6 \%)$, the mean age was 22.3 years (SD \pm 15.1 ), and about half were female ( $n=1,038 ; 51.8 \%)$. The majority of the participants had a secondary school education $(n=1,368 ; 68.3 \%)$ and a medium social economic status $(n=1,853 ; 92.5 \%)$ (Table 1). Of the 2,004 participants who enrolled, 1,989 (99.3\%) completed the 2-year follow-up study. A total of 15 participants were withdrawn: two participants because of noncompliance with protocol requirements (one participant was unavailable for study visits from June 14,2018 , and one participant could not be reached as of December 12, 2018); and 13 participants because they moved from the study area (Table 1). There was a total number of $3,869.3$ person-years of follow-up for the 2,004 participants (Table 2).

Most AFI episodes were because of non-LCD. During the 2-year follow-up, 251 AFI episodes were reported. Based on the RT-PCR test, 55 of these were because of LCD, 183 were because of non-LCD (Table 2), and 13 episodes were undetermined. Of the 13 episodes with undetermined results, three occurred in participants $<5$ years of age, five in participants $5-11$ years of age, three in participants $12-17$ years of age, and two in participants $\geq 18$ years of age (Table 2). The AFI cause remained undetermined when no validated PCR results were available.

Incidence rate of dengue (AFI because of LCD). A total of $55 \mathrm{AFI}$ episodes as a result of LCD were reported over the 2-year study period, of which two episodes were reported in one individual. The incidence rate of AFI because of LCD was 14.2 per 1,000 person-years (95\% Cl: $10.71-18.50)$ in the total cohort (Table 2). The incidence rate was the highest in children $5-11$ years of age at 22.7 per 1,000 person-years
(95\% Cl: 13.66-35.44) and children $<5$ years of age at 21.3 per 1,000 person-years $(95 \% \mathrm{Cl}$ : $10.61-38.02)$, while it was the lowest in adults $\geq 18$ years of age at 9.2 per 1,000 person-years (95\% Cl: 5.42-14.47) (Table 2).

Incidence rate of AFI because of non-LCD. A total of $183 \mathrm{AFI}$ episodes as a result of non-LCD were reported over the 2-year study period. No additional testing was performed to identify the cause of AFI in the non-LCD cases. The incidence rate of AFI because of non-LCD was 47.3 per 1,000 person-years (95\% Cl: $40.69-54.67)$ in the total cohort. The incidence rate was highest in children $<5$ years of age at 104.3 per 1,000 person-years (95\% Cl: $78.36-136.11)$ and lowest in adults at 17.3 per 1,000 person-years $(95 \% \mathrm{Cl}$ : 11.98-24.17) (Table 2).

Most LCD episodes were caused by DENV-2. Of the 55 LCD episodes, $83.6 \%(n=46)$ were caused by DENV-2, $10.9 \%(n=6)$ were because of DENV-1, and $5.5 \%(n=3)$ were because of DENV-3. The DENV-4 episodes were not reported. The incidence rate per 1,000 person-years was 1.6 for DENV-1 (95\% Cl: 0.57-3.38), 11.9 for DENV-2 (95\% Cl: 8.71-15.86), 0.8 for DENV-3 (95\% Cl: $0.16-2.27$ ), and 0.0 for DENV-4 (95\% Cl: 0.00-0.95) (Supplemental Table 2).

Symptoms of AFI because of LCD. During the 7-day follow-up period after the onset of an AFI because of LCD, the most commonly reported symptoms of interest (i.e., those occurring in at least $70.0 \%$ of the AFI episodes) included headache (over $90.0 \%$ of episodes on any given day), fatigue, myalgia, loss of appetite, and arthralgia (Table 3). The incidence of each of these symptoms remained quite stable throughout the 7-day follow-up, with similar incidences from Day 1 through Day 7. Bleeding was reported in less than $8.0 \%$ of episodes (4/55 episodes) (Table 3 ).

Symptoms of grade 3 intensity of AFI because of LCD. The most common symptoms with grade 3 intensity during the 7-day follow-up period, which prevented

TABLE 1

Demographic and households' characteristics (total cohort)

\begin{tabular}{lr}
\hline \multicolumn{1}{c}{ Demographic characteristic } & Participants \\
\hline$=2,004$ \\
\hline Age at enrollment, mean \pm SD in years & $22.3 \pm 15.1$ \\
Median in years (range) & $19.0(6$ months-49 years) \\
Age groups, $n$ (\%) & $257(12.8)$ \\
$<5$ years of age & $433(21.6)$ \\
$5-11$ years of age & $281(1.0)$ \\
$12-17$ years of age & $1,033(51.6)$ \\
$\geq 18$ years of age & $1,038(51.8)$ \\
Female gender, $n$ (\%) & $2(0.1)$ \\
Education, $n$ (\%) & $359(17.9)$ \\
None & $1,368(68.3)$ \\
Primary school & $24(1.2)$ \\
Secondary school & $7(0.3)$ \\
Graduate & $243(12.1)$ \\
Postgraduate & $1(0.05)$ \\
Not applicable (for participants $<5$ years of age) & $136(6.8)$ \\
Missing & $1,853(92.5)$ \\
Social Economic Status, $n(\%)$ & $15(0.7)$ \\
$\quad$ Low & $2(0.1)$ \\
Medium & $13(0.6)$ \\
Wigh & \\
$\quad$ Noncompliance with protocol requirements & \\
Moved from the study area & \\
\hline
\end{tabular}

$N=$ total number of participants; $n=$ number of participants in a given category. 
TABLE 2

Incidence rate of AFI because of LCD or non-LCD overall and by age group

\begin{tabular}{|c|c|c|c|c|c|c|c|c|}
\hline \multirow[b]{2}{*}{ Age group } & \multirow[b]{2}{*}{ N } & \multirow[b]{2}{*}{ Person-years } & \multicolumn{2}{|r|}{ AFI because of LCD } & \multirow[b]{2}{*}{$n$} & \multirow{2}{*}{$\begin{array}{c}\text { AFI because of non-LCD } \\
\begin{array}{c}\text { Incidence rate per } 1,000 \\
\text { person-years ( } 95 \% \mathrm{Cl}: \mathrm{LL}-\mathrm{UL})\end{array}\end{array}$} & \multirow[b]{2}{*}{$n$} & \multirow{2}{*}{$\begin{array}{c}\text { AFI because of } \\
\text { undetermined cause } \\
\text { Incidence rate per } 1,000 \\
\text { person-years }(95 \% \mathrm{Cl} \text { : } \\
\text { LL-UL) }\end{array}$} \\
\hline & & & $n$ & $\begin{array}{l}\text { Incidence rate per } 1,000 \\
\text { person-years (95\% Cl: } \\
\text { LL-UL) }\end{array}$ & & & & \\
\hline$<5$ years of age & 257 & 517.66 & 11 & $21.25(10.61-38.02)$ & 54 & $104.31(78.36-136.11)$ & 3 & $5.80(1.20-16.94)$ \\
\hline 5 to 11 years of age & 433 & 837.25 & 19 & $22.69(13.66-35.44)$ & 71 & $84.8(66.23-106.97)$ & 5 & 5.97 (1.94-13.94) \\
\hline 12 to 17 years of age & 281 & 548.47 & 7 & $12.76(5.13-26.30)$ & 24 & $43.76(28.04-65.11)$ & 3 & $5.47(1.13-15.98)$ \\
\hline$\geq 18$ years of age & 1,033 & $1,965.88$ & 18 & $9.16(5.43-14.47)$ & 34 & $17.3(11.98-24.17)$ & 2 & $1.02(0.12-3.68)$ \\
\hline Overall & 2,004 & $3,869.26$ & $55^{\star}$ & $14.21(10.71-18.50)$ & 183 & $47.3(40.69-54.67)$ & 13 & $3.36(1.79-5.75)$ \\
\hline
\end{tabular}

$\mathrm{AFI}=$ acute febrile illness; Incidence rate per 1,000 person-years = number of first event per 1,000 person-years; LCD $=$ laboratory confirmed dengue; LL $=$ lower limit; $N=$ number of participants at risk during the follow-up period; $n=$ number of first events reported during the follow-up period at risk; Person-years $=$ the sum of the years of observation of all participants within the age category expressed in years, censored at the first event; UL = upper limit.

${ }^{*}$ Of which two episodes were reported in one individual.

normal daily activities such as attending school or work were fatigue (25.5\%), headache/irritability (20.0\%), myalgia (18.2\%), arthralgia (14.6\%), and loss of appetite (14.6\%). The incidence of each of the grade 3 signs and symptoms remained stable throughout the 7-day follow-up (Table 4).

Signs and symptoms of AFI because of LCD, by age group. During the 7-day follow-up period after the onset of an AFI because of LCD, signs and symptoms of interest were almost identical from Day 1 through Day 7 in all age groups. The only variation was that one adult who had a headache/irritability on Day 1 to Day 5 did not report this symptom on Day 6 and Day 7 (Table 5).

There were no clinically important differences in the reporting profile of signs and symptoms across age groups. Similar to the signs and symptoms reported in the total cohort, headache/irritability, myalgia, and fatigue or decrease in everyday activity, were the most frequently reported signs and symptoms in each of the age groups, as they each occurred in more than $70.0 \%$ of AFI because of LCD. In all age groups, except those $<5$ years of age, arthralgia also occurred in more than $70.0 \%$ of episodes. In addition, in all groups $<18$ years of age, loss of appetite and reduced fluid intake occurred in more than $70.0 \%$ of the episodes. Vomiting was only common in the $<5$ years of age group. Rash and bleeding were least reported in all age groups. A rash was observed in 5 (16.7\%) episodes in children $<12$ years of age and in none of the 25 episodes in teenagers and adults. Bleeding was observed in $2(6.7 \%)$ of

TABLE 3

Symptoms of any intensity with AFI because of LCD during the 7-day period from the onset of fever

\begin{tabular}{|c|c|c|c|c|}
\hline \multirow[b]{2}{*}{ Symptoms } & \multicolumn{2}{|c|}{ Day 1-5 } & \multicolumn{2}{|c|}{ Day 6-7 } \\
\hline & $n$ & $\%$ & $n$ & $\%$ \\
\hline Headache/irritability & 51 & 92.73 & 50 & 90.91 \\
\hline Fatigue/decrease in everyday activity & 48 & 87.27 & 48 & 87.27 \\
\hline Myalgia (muscle pain) & 47 & 85.45 & 47 & 85.45 \\
\hline Loss of appetite & 43 & 78.18 & 43 & 78.18 \\
\hline Arthralgia (joint pain) & 42 & 76.36 & 42 & 76.36 \\
\hline Eye pain & 34 & 61.82 & 34 & 61.82 \\
\hline Reduced fluid intake & 33 & 60.00 & 33 & 60.00 \\
\hline Abdominal pain & 31 & 56.36 & 31 & 56.36 \\
\hline Nausea & 29 & 52.73 & 29 & 52.73 \\
\hline Vomiting & 29 & 52.73 & 29 & 52.73 \\
\hline Rash & 5 & 9.09 & 5 & 9.09 \\
\hline Any bleeding (skin, mouth, anus) & 4 & 7.27 & 4 & 7.27 \\
\hline
\end{tabular}

the episodes in children $<12$ years, in none of the teenagers, and in $2(11.1 \%)$ of the adults (Table 5).

Signs and symptoms of grade 3 intensity of AFI because of LCD, by age group. The incidence of symptoms with grade 3 intensity by age group did not change throughout the 7-day follow-up (Supplemental Table 3). Among the 11 episodes of AFI in the $<5$ years of age group, one case was reported, each of grade 3 , for headache/irritability, rash, loss of appetite, and reduced fluid intake. Among the 19 episodes in the 5-11 years of age group, four to five cases were reported, each of grade 3, for fatigue, headache/ irritability, myalgia, and loss of appetite. Among the seven episodes in the 12-17 years of age group, one case was reported, each of grade 3, for headache/irritability, arthralgia, loss of appetite, and fatigue/decrease in everyday activity. Among the 18 episodes in the $\geq 18$ years of age, eight cases of grade 3 fatigue were reported, six cases for myalgia, six cases for arthralgia, and five cases for headache/irritability (Supplemental Table 3).

Signs and symptoms of AFI because of non-LCD. Of the $183 \mathrm{AFI}$ episodes because of non-LCD, the most commonly reported signs and symptoms of interest (occurring in at least $70.0 \%$ of cases) during the 7-day follow-up period included, in order of frequency: headache/irritability $(n=159)$, fatigue/decrease in everyday activity $(n=152)$, loss of appetite ( $n=140)$, and myalgia $(n=133)$. As with AFI episodes because of LCD, the frequency of signs and symptoms reported in AFI episodes because of non-LCD were similar from Day 1 through Day 7 (Supplemental Table 4).

TABLE 4

Symptoms of grade 3 intensity with AFI because of LCD during the 7-day period from the onset of fever

\begin{tabular}{lcc}
\hline & \multicolumn{2}{c}{ Day $1-7$} \\
\cline { 2 - 3 } \multicolumn{1}{c}{ Symptoms } & $n$ & $\%$ \\
\hline Fatigue/decrease in everyday activity & 14 & 25.45 \\
Headache/irritability & 11 & 20.00 \\
Myalgia (muscle pain) & 10 & 18.18 \\
Arthralgia (joint pain) & 8 & 14.55 \\
Loss of appetite & 8 & 14.55 \\
Reduced fluid intake & 4 & 7.27 \\
Eye pain & 2 & 3.64 \\
Abdominal pain & 2 & 3.64 \\
Vomiting & 1 & 1.82 \\
Rash & 1 & 1.82 \\
\hline AFI $=$ acute febrile illness; LCD $=$ laboratory confirmed dengue; $n=$ number of \\
AFI episodes presenting with a sign or symptom of grade 3 intensity in a given category; $\%=$ \\
$n / 55 \times 100$.
\end{tabular}


TABLE 5

Signs and symptoms of any intensity with AFI because of LCD during the 7-day period from the onset of fever by age group

\begin{tabular}{|c|c|c|c|c|c|c|c|c|c|c|c|c|c|c|}
\hline \multirow[b]{3}{*}{ Symptoms } & \multicolumn{3}{|c|}{$<5$ years of age } & \multicolumn{3}{|c|}{$5-11$ years of age } & \multicolumn{3}{|c|}{$12-17$ years of age } & \multicolumn{5}{|c|}{$\geq 18$ years of age } \\
\hline & \multicolumn{3}{|c|}{ Day 1-7 } & \multicolumn{3}{|c|}{ Day 1-7 } & \multicolumn{3}{|c|}{ Day $1-7$} & \multicolumn{3}{|c|}{ Day 1-5 } & \multicolumn{2}{|c|}{ Day 6-7 } \\
\hline & N & $n$ & $\%$ & N & $n$ & $\%$ & N & $n$ & $\%$ & N & $n$ & $\%$ & $n$ & $\%$ \\
\hline Headache/irritability & 11 & 9 & 81.82 & 19 & 17 & 89.47 & 7 & 7 & 100.00 & 18 & 18 & 100.00 & 17 & 94.44 \\
\hline Eye pain & 11 & 7 & 63.64 & 19 & 12 & 63.16 & 7 & 5 & 71.43 & 18 & 10 & 55.56 & 10 & 55.56 \\
\hline Myalgia (muscle pain) & 11 & 10 & 90.91 & 19 & 17 & 89.47 & 7 & 5 & 71.43 & 18 & 15 & 83.33 & 15 & 83.33 \\
\hline Arthralgia (joint pain) & 11 & 6 & 54.55 & 19 & 14 & 73.68 & 7 & 6 & 85.71 & 18 & 16 & 88.89 & 16 & 88.89 \\
\hline Abdominal pain & 11 & 7 & 63.64 & 19 & 8 & 42.11 & 7 & 5 & 71.43 & 18 & 11 & 61.11 & 11 & 61.11 \\
\hline Nausea & 11 & 6 & 54.55 & 19 & 11 & 57.89 & 7 & 4 & 57.14 & 18 & 8 & 44.44 & 8 & 44.44 \\
\hline Vomiting & 11 & 8 & 72.73 & 19 & 11 & 57.89 & 7 & 4 & 57.14 & 18 & 6 & 33.33 & 6 & 33.33 \\
\hline Rash & 11 & 2 & 18.18 & 19 & 3 & 15.79 & 7 & 0 & 00.00 & 18 & 0 & 00.00 & 0 & 00.00 \\
\hline Any bleeding (skin, mouth, anus) & 11 & 1 & 9.09 & 19 & 1 & 5.26 & 7 & 0 & 00.00 & 18 & 2 & 11.11 & 2 & 11.11 \\
\hline Loss of appetite & 11 & 9 & 81.82 & 19 & 16 & 84.21 & 7 & 6 & 85.71 & 18 & 12 & 66.67 & 12 & 66.67 \\
\hline Fatigue/decrease in everyday activity & 11 & 10 & 90.91 & 19 & 17 & 89.47 & 7 & 6 & 85.71 & 18 & 15 & 83.33 & 15 & 83.33 \\
\hline Reduced fluid intake & 11 & 8 & 72.73 & 19 & 14 & 73.68 & 7 & 5 & 71.43 & 18 & 6 & 33.33 & 6 & 33.33 \\
\hline
\end{tabular}

The signs and symptoms did not show any clinically important differences among age groups. Among the 54 episodes of AFI because of non-LCD in the $<5$ years of age group, $>$ $70.0 \%$ reported headache/irritability, loss of appetite, and fatigue/decrease in everyday activity. Among the 71 episodes in the 5-11 years of age group and the 34 episodes in the $\geq$ 18 years of age group, $>70.0 \%$ reported headache/irritability ( $n=64 ; n=32$, respectively), myalgia ( $n=51 ; n=31$, respectively), loss of appetite ( $n=56 ; n=24$, respectively), and fatigue/decrease in everyday activity $(n=60 ; n=28$, respectively). In the $\geq 18$ years of age also arthralgia was reported in $>70.0 \%(n=31)$. Among the 24 episodes in the 12-17 years of age group, $>70.0 \%$ reported headache/irritability $(n=20)$, myalgia $(n=17)$, and fatigue/decrease in everyday activity $(n=17)$. Rash and any bleeding were least reported in all age groups (Supplemental Table 5).

Signs and symptoms of grade 3 intensity of AFI because of non-LCD. Symptoms with grade 3 intensity occurred in less than $10.0 \%$ of the $183 \mathrm{AFI}$ episodes because of non-LCD. Most frequently reported were fatigue/ decrease in everyday activity $(n=17)$ and headache/irritability $(n=16)$ (Supplemental Table 6).

The incidence of grade 3 intensity signs and symptoms by age group did not change throughout the 7-day follow-up. Among the 54 episodes of AFI because of non-LCD in the $<5$ years of age group, the most frequently reported symptoms were loss of appetite $(n=5)$, headache/irritability $(n=4)$, fatigue/decrease in everyday activity $(n=4)$, and reduced fluid intake $(n=4)$ at about $7.0-9.0 \%$ each. Among the 71 episodes in the 5-11 years of age group, the most frequently reported symptoms were fatigue/decrease in everyday activity $(n=8)$, headache/irritability $(n=7)$, myalgia $(n=5)$, and loss of appetite $(n=5)$ at about $7.0-11.0 \%$ each. Among the 24 episodes in the 12-17 years of age group, the most frequently reported symptoms were myalgia $(n=2)$, eye pain $(n=1)$, abdominal pain $(n=1)$, and nausea $(n=1)$ at about $4.0-8.0 \%$ each. Among the 34 episodes in the $\geq 18$ years of age, the most frequently reported symptoms were headache/irritability $(n=5)$, arthralgia $(n=7)$, myalgia $(n=6)$, and fatigue $(n=5)$ at about 14.0-20.0\% each (Supplemental Table S7).

Signs and symptoms associated with AFI because of LCD. Of the signs and symptoms in the $251 \mathrm{AFI}$ episodes, eye pain $(P<0.01)$, arthralgia $(P<0.05)$, and vomiting $(P<$
0.05) were found to be associated with AFI because of LCD in a univariate logistic regression, whereas only eye pain was associated in a multivariate logistic regression $(P<0.01)$ (Supplemental Table 8).

Hospitalizations. Of the 55 episodes of dengue, eight $(14.5 \%)$ episodes resulted in hospitalization, two $(3.6 \%)$ episodes in the 5-11 years of age group, 2 (3.6\%) episodes in the 12-17 years of age group, and 4 (7.3\%) episodes in the adult group. Of the 183 episodes of AFI because of non-LCD, seven (3.8\%) episodes resulted in hospitalization, three (1.6\%) episodes in the $<5$ years of age group, three (1.6\%) episodes in the $5-11$ years of age group, and one $(0.5 \%)$ episode in the adult group (Supplemental Table 9).

\section{DISCUSSION}

This prospective 2-year cohort study conducted in the Colombo district of Sri Lanka detected an incidence of LCD of 14.2 per 1,000 person-years in the total study population, consisting of adults and children. Incidence varied between age groups from 9.2 per 1,000 person-years in adults $\geq 18$ years of age to 22.7 per 1,000 person-years in children 5-11 years of age. The vast majority of the episodes $(n=46)$ were because of DENV-2, indicating this study was conducted during a DENV-2 dengue epidemic.

The dengue incidence rate we found (14.2 per 1,000 person-years) was comparable to the reported rate of suspected dengue in 2017 for the Colombo district, which was 1,419 per 100,000 population. $^{9}$ In 2017 , one of the worst outbreaks of dengue occurred in Sri Lanka; in all 25 districts, dengue incidence was significantly higher than in the previous 5 years (2012-2016). In the Colombo district, where our current study was performed, the incidence was 2.8-fold higher in 2017 than in the previous 5 years. ${ }^{9}$ Also, according to the International Federation of Red Cross and Red Crescent Societies, the outbreak of 2017 was the largest outbreak in three decades in Sri Lanka, with a total of 186,101 suspected dengue cases reported. ${ }^{10}$

Few reports of prospective studies of dengue incidence using active surveillance or even seroprevalence studies are available in Sri Lanka, especially for adults. When comparing seroprevalence data in children $<16$ years of age between 


\section{Plain Language Summary}

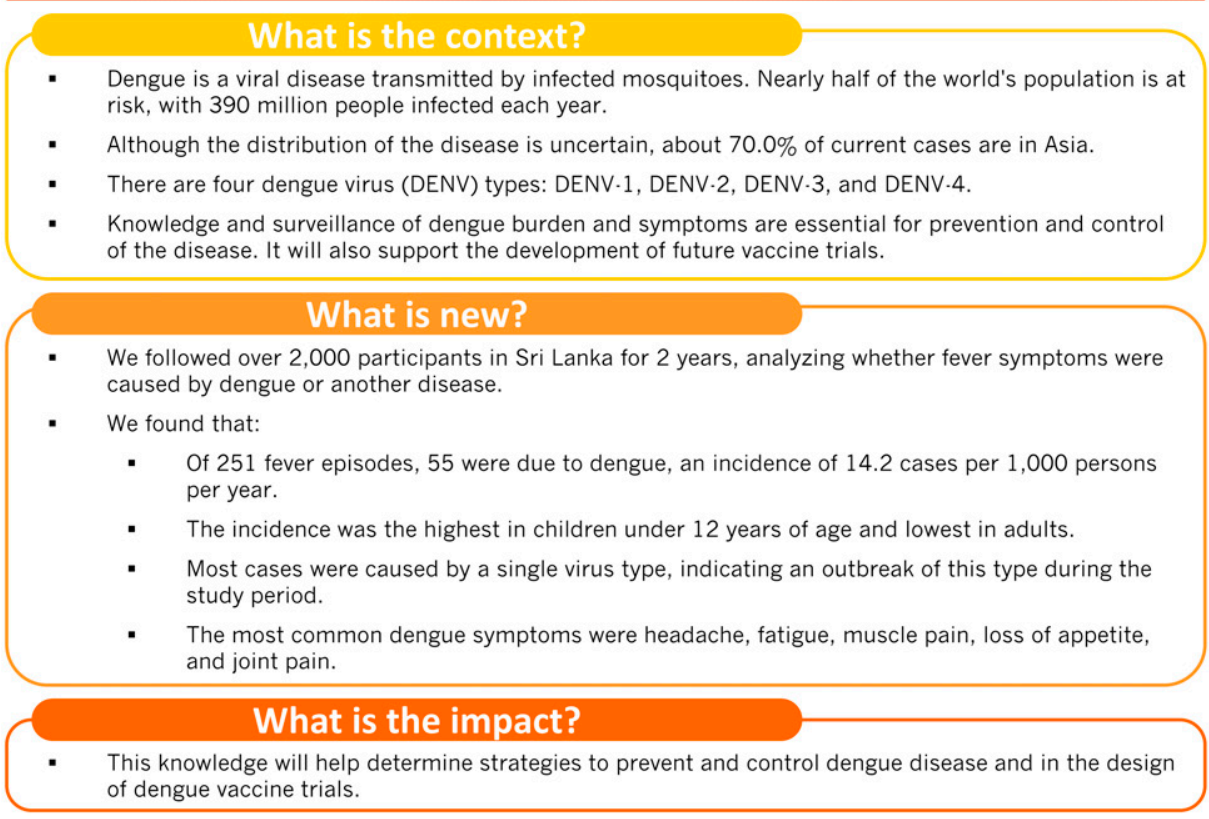

FIGURE 2. Plain language summary. This figure appears in color at www.ajtmh.org.

2003 and 2014, it was found that seroprevalence had significantly increased (from $34.1 \%$ to $50.7 \%$ ), and that the annual conversion rate was $1.5 \%$ in 2003 , while it was $3.8 \%$ in 2014. ${ }^{11}$ In another seroprevalence study, in about 800 children $<12$ years of age in an urban area in Sri Lanka from November 2008 to January 2009, more than half were already seropositive at enrollment, and the risk of primary infection was $14.0 \%$ per year. ${ }^{12}$ In a subsequent follow-up from November 2008 to January 2010, the incidence of clinically apparent LCD was 33.8 per 1,000 person-years $(95 \%$ $\mathrm{Cl}$ : 22.4-48.8). ${ }^{13}$ Although their diagnosis method of LCD was not the same as in our study, their incidence of clinically apparent LCD was somewhat higher but comparable to the incidence we observed in children $<12$ years of age, which was 22.1 per 1,000 person-years on average. In our study, LCD was based on a positive RT-PCR test, while in the 2008-2010 study, LCD was based on a positive result in two of three diagnostic assays (RT-PCR, increasing levels of IgM or IgG in paired acute phase, and convalescent-phase blood samples), which likely increased their number of positive results. ${ }^{13}$ The total dengue incidence in the 2008-2010 study was 83.9 per 1,000 person-years; as for this incidence, in addition to clinically apparent LCD cases, children who had clinically inapparent dengue and seroconverted during the year were counted.

In Sri Lanka, dengue is endemic, and from 1991-2008 dengue epidemics occurred every few years on the background of the endemic transmission. ${ }^{14}$ Since then, dengue cases steadily increased according to data from the passive national surveillance system. ${ }^{15}$ Dominant serotypes varied throughout the years, as DENV-3 was the dominant strain from $1981-1998,{ }^{16}$ both DENV-2 and DENV-3 were common in 2003-2006, ${ }^{17}$ and DENV-1 was the dominant serotype from $2006-2016 .^{18-20}$ The large majority (83.6\%) of LCD episodes in our study was because of DENV-2, suggesting a DENV-2 epidemic. This observation was recently confirmed in a virologic study, which showed that in 2017, during the largest dengue epidemic in Sri Lanka so far, DENV-2 was at $89.0 \%$ the dominant serotype. ${ }^{9}$

The clinical challenges of distinguishing dengue-related AFI from AFI because of other causes are well documented. ${ }^{18}$ Even the improved guidelines for the diagnosis of dengue by the $\mathrm{WHO}$ based on clinical criteria ${ }^{1}$ were found to have low specificity $(65.0 \%)$ to accurately diagnose dengue when analyzed in a prospective study of patients hospitalized with fever in Sri Lanka during a DENV-1 epidemic. ${ }^{18} \mathrm{We}$ also found that the signs and symptoms of interest were indeed quite similar between LCD and non-LCD cases, as both groups reported headache, fatigue, myalgia, and loss of appetite in more than $70.0 \%$ of cases. Only arthralgia was a symptom of interest for LCD but not for non-LCD, although in the non-LCD group it was also present in $64.5 \%(n=$ 118). The most common symptoms of LCD with grade 3 intensity were fatigue (25.5\%), headache/irritability $(20.0 \%)$, myalgia (18.2\%), arthralgia (14.6\%), and loss of appetite (14.6\%). The most common symptoms with grade 3 intensity because of non-LCD were fatigue (9.3\%) and headache/irritability (8.7\%). In our cohort, eye pain, arthralgia, and vomiting were associated with AFI because of LCD in a univariate regression analysis, although only eye pain was also associated in a multivariate regression analysis. Interestingly, although previously eye pain has been found to be a common clinical feature of symptomatic dengue, ${ }^{21}$ it is not in the current WHO guidelines for the diagnosis of dengue. ${ }^{1}$ The 
explanation for this may be that eye pain is more common in younger adult (18-35 years of age) than in older adult $(\geq 36$ years of age) dengue patients, ${ }^{22}$ while the $\mathrm{WHO}$ guidelines do not differentiate by age. ${ }^{1}$ Overall, it is not possible to distinguish between LCD and non-LCD based on clinical presentation alone. This emphasizes the need for laboratory diagnosis to distinguish between the causes of AFI, as knowing the cause of the AFI allows for appropriate treatment at the individual level and targeted prevention at the population level.

One study limitation was that our dengue definition was based on $\geq 2$ days of AFI regardless of other dengue symptoms. This definition is problematic as few participants would undergo $\geq 2$ days of fever without self-administering antipyretics, and parents would very likely administer them to their children. In addition, we know from a large study assessing dengue presentation that, although only a low percentage of dengue patients present without fever $(3.4 \%$ of 14,756 patients), the large majority of those did have hemorrhagic manifestations (65.7\%), and a substantial number of them had failure of at least one organ (30.7\%). ${ }^{23}$ Also, as discussed earlier, a substantial number of clinically inapparent cases will be missed. Another limitation of the study is the missed opportunity to identify the cause of the AFI in non-LCD cases. It would have made the study more informative to both health authorities and researchers and clinicians interested in AFI in Sri Lanka in general.

In conclusion, this study occurred during a dengue epidemic because of DENV-2. A total of 55 LCD episodes were found, mostly in children $\leq 11$ years, and incidence was 14.2 per 1,000 person-years, ranging from 9.2 to 22.7 per 1,000 person-years depending on age. Our study contributes to the current knowledge of the epidemiology of dengue infection in Sri Lanka. This knowledge will help inform surveillance strategies as well as the design of dengue vaccine efficacy trials in Southeast Asia. The findings of this study are summarized in plain language in Figure 2.

Received May 28, 2021. Accepted for publication August 12, 2021.

Published online November 1, 2021.

Note: Supplemental tables appear at www.ajtmh.org.

Acknowledgments: We would like to thank the participants and their legal representatives for their participation in the study. The authors thank all of the field and laboratory technical staff who helped with participant recruitment and follow-up, sample processing, and testing, as well as the regulatory and administrative staff who helped during the study and the global dengue purified inactivated vaccine (DPIV) program team. We also want to thank the following persons who contributed to the study: Adrienne Guignard, Meng Shi, Lucian Jayasuria, Vinaykumar Nandimath, and Anand Harpalani. We also thank the Business \& Decision Life Sciences platform for editorial assistance and manuscript coordination on behalf of GSK. Janne Tys (Business \& Decision Life Sciences on behalf of GSK) coordinated the manuscript development and editorial support. Esther van de Vosse provided medical writing support.

Funding support: GlaxoSmithKline Biologicals SA funded this study (NCT02570152) and was involved in all stages of study conduct, including analysis of the data. GlaxoSmithKline Biologicals SA also covered all costs associated with the development and publication of this manuscript.

Disclosures: The institution of H. T. and P. S. was funded by the GSK group of companies to conduct the study. P. S. received personal fees by the Ministry of Health for the position of co-investigator. M. d. B., S. G., L. M., S. M., and V. V. are employees of the GSK group of companies, and M. d. B., L. M., and S. M. also hold shares in the GSK group of companies. R. P. and A. S. were employees of the GSK group of companies and held shares in the GSK group of companies at the time of study conduct. P. P. has no conflict of interest. All authors declare no other financial and non-financial relationships and activities. All authors participated in the design or implementation or analysis, and interpretation of the study, and the development of this manuscript. All authors had full access to the data and gave their final approval before submission.

Authors' addresses: Hasitha Tissera and Paba Palihawadana, Epidemiology Unit, Ministry of Health, Colombo, Sri Lanka, E-mails: dr korelege@yahoo.co.uk and dr.paba@gmail.com. Preshila Samaraweera, National Dengue Control Unit, Ministry of Health, Colombo, Sri Lanka, E-mail: preshilas@gmail.com. Melanie de Boer, GSK, Rockville, MD, E-mail: melanie.ann.de.boer@gmail.com. Robert Paris, Moderna, Cambridge, MA, E-mail: robparis2003@ yahoo.com. Alexander Schmidt, Bill \& Melinda Gates Medical Research Institute, Cambridge, MA, E-mail: as337y@gmail.com. Sanjay Gandhi, GSK, GSK House, Worli, Mumbai, India, E-mail: sanjay.t.gandhi@gsk.com. Ludovic Malvaux, Fleming Ave, 20, Wavre, Belgium, E-mail: ludovic.x.malvaux@gsk.com. Valerie Vantomme, GSK, Rixensart, Belgium, E-mail: valerie.vantomme@ gsk.com. Shailesh Mehta, GSK, Wavre, Belgium, E-mail: shailesh.i. mehta@gsk.com.

This is an open-access article distributed under the terms of the Creative Commons Attribution (CC-BY) License, which permits unrestricted use, distribution, and reproduction in any medium, provided the original author and source are credited.

\section{REFERENCES}

1. World Health Organization, 2009. Dengue Guidelines for Diagnosis, Treatment, Prevention and Control: New Edition. Report No.: WHO/HTM/NTD/DEN/2009.1. Geneva, Switzerland: WHO. Available at: https://apps.who.int/iris/bitstream/handle/ 10665/44188/9789241547871_eng.pdf. Accessed February 2, 2021.

2. Stanaway JD et al., 2016. The global burden of dengue: an analysis from the Global Burden of Disease Study 2013. Lancet Infect Dis 16: 712-723.

3. World Health Organization, 2020. Dengue and Severe Dengue. Geneva, Switzerland: WHO. Available at: https://www.who.int/ news-room/fact-sheets/detail/dengue-and-severe-dengue. Accessed February 2, 2021.

4. World Health Organization, 2020. Global Strategy for Dengue Prevention and Control. 2012-2020. Geneva, Switzerland: WHO. Available at: https://apps.who.int/iris/bitstream/handle/ 10665/75303/9789241504034_eng.pdf. Accessed February 2, 2021.

5. Brady OJ, Gething PW, Bhatt S, Messina JP, Brownstein JS, Hoen AG, Moyes CL, Farlow AW, Scott TW, Hay SI, 2012. Refining the global spatial limits of dengue virus transmission by evidence-based consensus. PLoS Negl Trop Dis 6: e1760.

6. Bhatt $S$ et al., 2013. The global distribution and burden of dengue. Nature 496: 504-507.

7. International Council for Harmonization, 2016. ICH Harmonised Guideline Integrated Addendum to ICH E6(R1): Guideline for Good Clinical Practice ICH E6(R2) ICH Consensus Guideline. Geneva, Switzerland: ICH. Available at: https://database.ich. org/sites/default/files/E6_R2_Addendum.pdf. Accessed April 26, 2021.

8. U.S. National Library of Medicine, 2015. A Cohort Study to Determine the Incidence of Dengue Fever and to Build Capacity for Dengue Vaccine Trials in Dengue-endemic Regions of South Asia. Available at: https://clinicaltrials.gov/ct2/show/ NCT02570152. Accessed February 9, 2021.

9. Tissera HA et al., 2020. Severe dengue epidemic, Sri Lanka, 2017. Emerg Infect Dis 26: 682-691.

10. International Federation of Red Cross and Red Crescent Societies (IFRC), 2018. Sri Lanka/Dengue DREF Final Report (MDRLK007). Report No.: MDRLK007. Geneva, Switzerland: IFRC. Available at: https://reliefweb.int/report/sri-lanka/sri-lankadengue-dref-final-report-mdrlk007. Accessed February 9, 2021. 
11. Jeewandara $C$ et al., 2015. Change in dengue and Japanese encephalitis seroprevalence rates in Sri Lanka. PLoS One 10: e0144799.

12. Tam CC, Tissera $H$, de Silva $A M$, de Silva $A D$, Margolis $H S$, Amarasinge $A, 2013$. Estimates of dengue force of infection in children in Colombo, Sri Lanka. PLoS Negl Trop Dis 7: e2259.

13. Tissera $H$, Amarasinghe $A$, de Silva $A D$, Kariyawasam $P$, Corbett KS, Katzelnick L, Tam C, Letson GW, Margolis HS, de Silva $\mathrm{AM}, 2014$. Burden of dengue infection and disease in a pediatric cohort in urban Sri Lanka. Am J Trop Med Hyg 91: 132-137.

14. Kulatilaka TA, Jayakuru WS, 1998. Control of dengue/dengue haemorrhagic fever in Sri Lanka. Dengue Bull 22: 53-61.

15. Ministry of Health - Epidemiology Unit - Sri Lanka, 2019. Distribution of Notification (H399). Dengue Cases by Month. Colombo, Sri Lanka: Ministry of Health. Available at: https://www.epid. gov.lk/web/index.php?option=com_casesanddeaths\&ltemid= 448\&lang=en\#. Accessed February 9, 2021.

16. Messer WB, Gubler DJ, Harris E, Sivananthan K, de Silva AM, 2003. Emergence and global spread of a dengue serotype 3, subtype III virus. Emerg Infect Dis 9: 800-809.

17. Kanakaratne N, Wahala WM, Messer WB, Tissera HA, Shahani A, Abeysinghe N, de Silva AM, Gunasekera M, 2009. Severe dengue epidemics in Sri Lanka, 2003-2006. Emerg Infect Dis 15: 192-199.

18. Bodinayake CK et al., 2018. Evaluation of the WHO 2009 classification for diagnosis of acute dengue in a large cohort of adults and children in Sri Lanka during a dengue-1 epidemic. PLoS Negl Trop Dis 12: e0006258.

19. Raut $R$ et al., 2019. Dengue type 1 viruses circulating in humans are highly infectious and poorly neutralized by human antibodies. Proc Natl Acad Sci USA 116: 227-232.

20. Tissera HA et al., 2011. New dengue virus type 1 genotype in Colombo, Sri Lanka. Emerg Infect Dis 17: 2053-2055.

21. Tomashek KM et al., 2017. Clinical and epidemiologic characteristics of dengue and other etiologic agents among patients with acute febrile illness, Puerto Rico, 2012-2015. PLoS Negl Trop Dis 11: e0005859.

22. Low JGH et al., 2011. The early clinical features of dengue in adults: challenges for early clinical diagnosis. PLoS Negl Trop Dis 5: e1191.

23. Tukasan C, Furlan NB, Estofolete CF, Nogueira ML, da Silva NS, 2017. Evaluation of the importance of fever with respect to dengue prognosis according to the $2009 \mathrm{WHO}$ classification: a retrospective study. BMC Infect Dis 17: 6. 